nach den Jahreszeiten, bleibt für die Sanatorien dagegen ziemlich ausgeglichen. Im Mittel beträgt er für den Winter und Sommer bis 70 und 80 , für die $Z$ wischenzeiten dagegen oft nur um 20 Prozent.

Für die Kinderheime, Institute und namentlich die Chalets darf man nun, auf Grund auch der Erfahrungen, die in Graubünden anhand einer etwas detaillierteren kantonalen Statistik gemacht worden sind, annehmen, daß das Jahresmittel des Ausnützungsgrades leicht höher liegt. Es wird so möglich, für die einzelnen Stationen wenigstens zu schätzen, wie hoch die Gesamtbelegung in den einzelnen Monaten ist. Es liegt auf der Hand, daß das Total der Logiernächte gegenüber der Zahl der Übernachtungen nur in Hotels, Pensionen und Sanatorien andere, zum Teil beträchtlich abweichende Werte ergibt. So ist Leysin nicht mit Abstand größter Fremdenplatz der Bergregion der Alpennordseite zwischen Rhone und Rhein; vielmehr machen sich bei solcher Betrachtungsweise im Gebiet der Berner und der Waadtländer Alpen Leysin, Saanen und Adelboden, dicht gefolgt von Grindelwald, den Rang streitig.

\title{
BENÜTZTE LITERATUR
}

Statistisches Jahrbuch der Schweiz, 1962. - Ergebnisse der Eidg. Volkszählungen 193o, 1950, 1960. - G. Menges: Möglichkeiten der Quantifizierung und Messung des Fremdenverkehrs. Zeitschrift für Fremdenverkehr, 15, 1960. - W. Hunziker und K. Krapf: Grundriß der Allgemeinen Fremdenverkehrslehre. Zürich 1942. - F. Rubli: Der Wintertourismus in der Schweiz; Entwicklung, Struktur und volkswirtschaftliche Bedeutung. Bern/Basel 1953. - M. Studer: Die Erschließung des Berner Oberlandes durch den Fremdenverkehr und ihre Auswirkungen auf Produktion und Wirtschaftsgesinnung. Schweiz. Beiträge zur Verkehrswissenschaft, Heft $27,1947$. - Germaine Veyret: La deuxième révolution économique et démographique des Alpes du Nord: Les sports d'hiver. Réflexions et suggestions. Revue de géographie alpine, XLVII 1959.

\author{
KLIMATOLOGIE UND METEOROLOGIE \\ AM ANFANG DES 2. JAHRHUNDERTS \\ REGELMÄSSIGER \\ SCHWEIZERISCHER WETTERBEOBACHTUNGEN
}

\author{
MAX SCHÜEPP
}

Am 1. Dezember 1963 waren hundert Jahre verflossen, seit das Netz schweizerischer meteorologischer Beobachtungen gegründet wurde. Kein Krieg hat in unserem Land einen Unterbruch der Serie gebracht. Keine politische Umwälzung, welche eine Gefahr für die Kontinuität der Methoden in bezug auf Zeit, Art und Verarbeitung der Messungen gebildet hätte, ereignete sich im Laufe dieser hundert Jahre. Wir besitzen somit ein großes und - wenn auch mit Mängeln behaftetes - im allgemeinen befriedigendes Beobachtungsmaterial. Wenn auf diese Weise sich ein Betrieb eingelebt hat, ist es notwendig, sich von Zeit zu Zeit darüber Rechenschaft zu geben, ob der Weg, der 1864 eingeschlagen wurde, noch richtig ist, ob wir ihn auch in den kommenden Jahren und Jahrzehnten weiter verfolgen sollen oder ob eine prinzipielle Anpassung an die neue Zeit notwendig ist.

Anlaß zu einem solchen Rück- und Ausblick bildet nicht nur das Jubiläum, auch die heutige Entwicklung von Technik und sozialer Stellung der Beobachter zwingt zu einer Standortbestimmung. Wir möchten daher in den nachfolgenden Zeilen versuchen, 
einen kurzen Überblick der heutigen Lage sowohl auf klimatologischer als auch synoptischer Seite zu geben, sind doch beide Arbeitsgebiete am Beobachtungsnetz interessiert, stellen aber verschiedene Ansprüche. Die Klimatologie ist dabei das konservative Element. Sie ist an möglichst unveränderten Aufstellungen, Beobachtungszeiten und Auswertungen interessiert. Jeder Wechsel muß gründlich überprüft werden, ob er einen wesentlichen Fortschritt bringt, welcher den erhöhten, durch die Reduktion der alten auf die neue Reihe bedingten Aufwand an Arbeit rechtfertigt. Die Synoptik, welche für den Augenblick und die Zukunft arbeitet, ist dagegen an jeder Veränderung interessiert, welche eine Verbesserung bringt, mag sie auch noch so klein sein. Es gilt somit, die beiden in mancher Beziehung entgegengesetzten Kräfte soweit als möglich zu einem harmonischen Ganzen zu vereinigen. Dies ist nur erreichbar durch teilweise Trennung der Beobachtungsnetze, wie sie sich im Laufe der letzten drei Jahrzehnte vor allem durch die Entwicklung des Flugwetterdienstes ergeben hat.

Betrachten wir zunächst den Stand der Klimatologie. Nach der Jahrhundertwende hat diese Abteilung der Meteorologie ihre Blütezeit erlebt, als genügend Jahre für eine Beurteilung der klimatologischen Verhältnisse in den verschiedenen Landesteilen vorhanden waren und 1909/10 eine erste Zusammenstellung der Ergebnisse im «Klima der Schweiz» von Maurer, Billwiller und Hess erschien. Seither sind schon mehr als 5 Jahrzehnte verflossen. Eine Neubearbeitung ist dringend notwendig geworden. Sie wurde zwar begonnen, konnte aber bisher nur zum kleinsten Teil fertiggestellt werden, da die Klimatologie in der Zwischenzeit durch die starke Entwicklung der anderen $Z$ weige der Meteorologie etwas in den Hintergrund gedrängt wurde und nicht die notwendigen personellen Hilfsmittel für eine rasche Beendigung der Arbeiten zur Verfügung standen. Die nächsten Jahre sollen jedoch in dieser Beziehung einen Wandel bringen, indem mehr und mehr die technischen Hilfsmittel der automatischen Datenverarbeitung mit Lochkarten eingesetzt werden. Das Programm für die Herausgabe einer neuen, die Jahrzehnte 1901-1960 umfassende Klimatologie der Schweiz ist aufgestellt. Es gliedert sich in drei Teile. Im ersten Band werden die Zahlentabellen für die einzelnen Wetterelemente zusammengestellt. Er wird die Grundlage bilden für den zweiten Band, der eine Darstellung der Verhältnisse in den einzelnen Landesteilen bringen wird, mit Text und graphischen Darstellungen, also gewissermaßen eine neue, auf die heutige Zeit bezogene Auflage des «Klima der Schweiz». Der dritte Band endlich wird die einzelnen Witterungslagen behandeln, welche in der Schweiz auftraten. Er möchte eine Brücke schlagen zwischen Klimatologie und Meteorologie, in einer neuen, in den früheren Jahrzehnten noch nicht üblichen Art, indem die einzelnen Witterungstypen für sich gesondert betrachtet werden in ihrer jahreszeitlichen Häufigkeit und in ihren charakteristischen Auswirkungen auf die Wettererscheinungen in den verschiedenen Landesgegenden. Der zweite Teil des 3. Bandes soll dem Zusammenhang zwischen den einzelnen für die Prognose wichtigen Wetterelementen (besonders Temperatur, Sonnenscheindauer sowie Bewölkung, Niederschlag und Wind) und den verschiedenen Witterungslagen gewidmet werden. Wir müssen ja immer mehr versuchen, aus dem Stadium der Klimatographie, der reinen Beschreibung der vorhandenen Verhältnisse, in dasjenige der Klimatologie, der Erklärung dieser Erscheinungen überzugehen. Das klimatologische Material muß helfen, unsere Kenntnis über den Witterungsablauf zu vertiefen. Dieser Forderung wird die Dreiteilung in Grundlagenmaterial, regionale und witterungsklimatologische Bearbeitung gerecht. Das Werk soll im Jahre 1971 fertiggestellt sein. Dann wird ein neues Jahrzehnt an Beobachtungen vorliegen, welches wie seine Vorgänger zunächst eine Überprüfung und nachfolgend eine Bearbeitung in einem Ergänzungsband erfordern wird. In den nächsten Jahren werden daher die Anstrengungen auf klimatologischem Gebiet auf dieses Werk gerichtet sein. Das vergangene Jahrzehnt war im wesentlichen der Verarbeitung gewidmet gewesen, mußten doch die Meldungen des klimatologischen Netzes für die Lochkartenauswertung vorbereitet werden. Wohl bedingte dies keine bedeutende Um- 


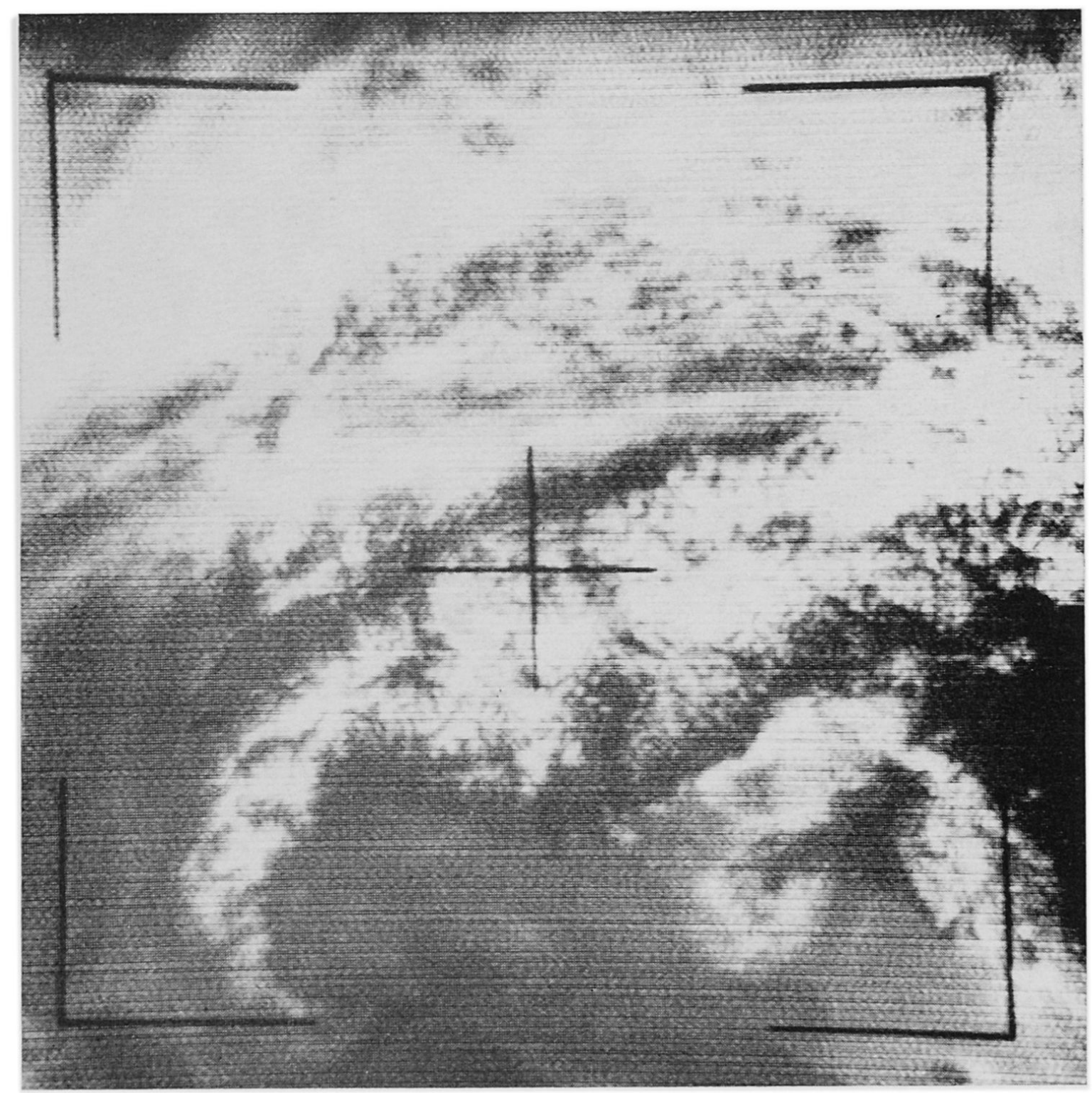

Diese vom Satelliten Tiros aus gemachte photographische Aufnahme zeigt die Wolkendecke über dem Alpengebiet am 21. Juni 1963, mittags. Der bewölkte Alpenkranz ist deutlich zu erkennen. Die Schweiz befindet sich im linken unteren Quadranten südwestlich des Mittelkreuzes.

Die großen Talkessel (Aostatal, Wallis, Rheintal, Etschgebiet) und der Alpennordrand zeigen starke Auflösung der Wolkenschicht. Der Mittelmeerraum um Turin-Mailand-Genua hat heiteren Himmel, während über Nordfrankreich und Mitteldeutschland eine große, nur stellenweise durchbrochene Decke liegt

stellung in der Art der Beobachtung und der Aufzeichnungen, aber eine große Instruktionsarbeit, bis ein einheitlicher Standard der Notizen erreicht war, welcher für eine Vergleichbarkeit der Resultate notwendig ist. Noch ist diese Forderung für einzelne Wetterelemente nicht völlig erreicht, speziell Feuchtigkeit, Nebel, Gewitter und Wind legen einer genauen Messung bzw. Beobachtung große Hindernisse in den Weg. Die verwendeten Haar-Hygrometer sind ungeau, die Lithium-Chlorid Hygrometer verlangen eine genaue Wartung, die Aspirationspsychrometer eine verhältnismäßig komplizierte Messung besonders im Winter, so daß im größten Teil des Netzes nach wie vor die Haar-Hygrometer verwendet werden müssen. Ähnliche instrumentelle Schwierigkei- 
ten ergeben sich bei den Windmessern und den Gewitterzählern, während beim Nebel die genaue Augenbeobachtung der 1-km-Sichtmarken schwierig auf einen einheitlichen Stand zu bringen ist. Es ist jedoch notwendig für eine Lochkartenauswertung, daß das Material nicht erst nach der eingehenden Bearbeitung für eine Klimatologie auf einen einheitlichen Standard gebracht, sondern schon vor der Lochung genau überprüft wird. Wohl sind die modernen elektronischen Datenverarbeitungsmaschinen - z. B. die IBM 7074 und 1401 des Eidg. Statistischen Amtes in Bern, welche die monatlichen und jährlichen meteorologischen Auswertungen besorgen - fähig, manche fehlerhaften Meldungen zu eruieren und auszumerzen, aber sie ersetzen doch das menschliche Gehirn mit der jahrelangen klimatologischen Erfahrung nicht. Dieses ist nach wie vor notwendig für die Überprüfung der Beobachtungen.

Die neue Zeit hat nicht nur die technische Entwicklung mit ihren Vorteilen gebracht, sie erschwerte gleichzeitig den Beobachtungsdienst durch die damit gekoppelte soziale Entwicklung. Wo findet sich heute ein einzelner Beobachter, der gegen ein bescheidenes Entgelt täglich 3mal 07.30, 13.30 und 21.30 Uhr werktags und sonntags seine Ablesungen durchführt, dazu noch in der Lage ist, in den Zwischenzeiten Nebel und Gewitter zu notieren? Noch ist es - mit zunehmenden finanziellen Aufwendungen - möglich, das Netz aufrechtzuerhalten, aber die Zeitspanne, die uns bleibt, bis sich infolge der veränderten Verhältnisse eine Automatisierung durchsetzen muß, wird kürzer und kürzer. Noch sind aber die entsprechenden Apparaturen nur zum Teil entwickelt, speziell die Bewölkungsmessung bereitet erhebliche Schwierigkeiten. Trotzdem muß ein weiterer Fortschritt in Richtung auf die automatische fernmeldende Station erzielt werden, wenn nicht infolge zunehmender personeller Schwierigkeiten eines Tages die Aufrechterhaltung des meteorologischen Netzes in Frage gestellt werden soll. Das zweite Jahrhundert wird somit sicher einen entscheidenden Wandel bringen, einen Fortschritt insofern, als ein Beobachtungsstandort nicht mehr wie bisher beim Tod eines Betreuers an einen andern Platz versetzt werden muß mit all den damit verbundenen Umtrieben und Nachteilen in der Bearbeitung. Zudem wird der Beobachtungsstandort frei wählbar sein, während er jetzt an die zufällige Behausung gebunden ist und vor allem die Windbeobachtungen dadurch nur in Ausnahmefällen wirklich gute, repräsentative Werte ergeben. Auch die Aufstellung der Sonnenscheinautographen bereitet oft erhebliche Schwierigkeiten. Bei beiden Elementen mußten daher bei einzelnen Stationen Registrierungen an abseits aufgestellten Standorten zu Hilfe genommen werden, was jedoch die Auswertung erschwert.

Dieser Abriß mag genügen, um die prinzipielle Lage und die notwendigen Umstellungen sowie die künftigen Aufgaben der schweizerischen Klimatologie zu skizzieren. Wir übergehen die speziellen Probleme der Witterungsklimatologie, welche sich noch in den «Kinderschuhen» befindet und erst in zwei bis drei Jahren in größerem Maßstab in Angriff genommen werden kann, und wenden uns nun der Synoptik zu, dem Gebiet, welches für viele Fernstehende gleichbedeutend ist mit Meteorologie, weil die Wetteranalysen und -prognosen den größten Widerhall und die weiteste Verbreitung in der Bevölkerung durch Radio und Fernsehen finden. Klimatologie und Forschung auf dem Gebiet der freien Atmosphäre sind weniger diskutierte Arbeitsgebiete, und der spezielle Flugwetterdienst ist wohl für die Aeronautik sehr wichtig, hat aber mit Ausnahme der damit gekoppelten Sturmwarnung für die breitere Öffentlichkeit weniger Bedeutung.

Die Synoptik kann zwar nicht auf einen hundertjährigen Bestand in der Schweiz zurückblicken, doch sind es immerhin 86 Jahre, seit die ersten Wetterkarten im Juni 1878 veröffentlicht wurden. Seither hat sich das synoptische Stationsnetz sowohl in die Breite als auch in die Höhe entwickelt. Es besteht zum Teil aus eigenen Meldestellen, zum Teil werden die Beobachtungen des klimatologischen Dienstes verwendet. Seit 30 Jahren haben sich die aerologischen Messungen hinzugesellt bis in immer größere Höhen hinauf mit der Einführung der Raketen und Satelliten. Nun ist diese Entwicklung nahezu abgeschlossen, es sind nur noch die - vor allem auf der Südhalbkugel der 
Erde bedeutenden - Lücken im Beobachtungsnetz zu schließen, besonders auf den Weltmeeren.

Die Grundlagen für die genauere Analyse und Prognose der Wetterabläufe sind somit vorhanden, es gilt nun, allmählich sich Einblick in das verwickelte Zusammenspiel aller wirksamen Faktoren zu verschaffen, wobei auf zwei verschiedenen Wegen vorgegangen werden kann. Einerseits lassen sich mit Hilfe der Strömungsgleichungen mathematisch unter vereinfachenden Annahmen die zu erwartenden Änderungen des Stromfeldes berechnen, vor allem für die mittleren Atmosphärenschichten, im Niveau um $5000 \mathrm{~m}$. Die modernen elektronischen Rechenmaschinen gestatten eine Berechnung der Prognosenkarten auf 2-3 Tage, teilweise auf 4 Tage hinaus, doch geben sie noch nicht bei allen Wetterlagen zuverläßige Resultate, und zudem ist damit erst die für den normalen Benützer meist nicht wichtige Höhenströmung bekannt. Ob das Wetter in Bodennähe kalt oder warm, trocken oder naß, sonnig oder bedeckt sei, muß zunächst weiterhin auf Grund der Erfahrung beurteilt werden. Die berechneten Karten haben aber den Vorteil, daß die Methoden im Laufe der Zeiten verbessert werden können. Nur dürfen wir keine raschen Fortschritte erwarten, das Zusammenspiel aller Faktoren ist so verwickelt, daß offenbar nur komplizierte mathematische Modellatmosphären die Verhältnisse auf längere Zeit mit genügender Annäherung wiedergeben können. Wir müssen daher mit Hilfe der Witterungsklimatologie aus ähnlichen früheren Fällen Schlüsse auf die kommende Entwicklung ziehen und allmählich versuchen, die jetzt noch nicht bekannten Einflüsse zu bestimmen. Theorie und Praxis müssen $\mathrm{Hand}$ in Hand gehen, wenn eine möglichst zuverläßige Prognose auf mehrere Tage hinaus aufgestellt werden soll. Während die Methoden der 1-2-Tage-Prognosen, welche zum größten Teil auf einer Extrapolation der bisherigen Entwicklung aufgebaut sind, sich voraussichtlich nur wenig ändern werden, ist bei den Mittel- und Langfristprognosen noch alles im Fluß bzw. steckt noch in den Kinderschuhen. Die kommenden Jahre sollen zeigen, was auf diesem für die Praxis sehr wichtigen Gebiet erreicht werden kann. Zunächst ist vor allem Forschungsarbeit notwendig, und es ist nicht anzunehmen, daß diese rasche Erfolge aufweisen wird. Wohl hat die Technik bedeutende Fortschritte in bezug auf Nachrichten- und Bildübermittlung gebracht, welche auch dem Wetterdienst zugute kommen - denken wir nur an die instruktiven Wolkenaufnahmen von den Satelliten aus -, aber es handelt sich bei den gewonnenen Auswertungen um Analysen der gegenwärtigen Lage, nicht um Prognosen. Wenn auch eine gute Analyse ein großer positiver Beitrag für eine gute Prognose darstellt, so ist sie doch nur eine günstige Ausgangsbasis und kein Resultat. Die Satelliten geben uns aber erstmals die Möglichkeit, dem ganzen Wettermechanismus an der Quelle nachzugehen, liegt doch die Steuerung letzten Endes mit großer Wahrscheinlichkeit bei der von der Sonne ausgehenden Strahlung, die wir am Grunde der Atmosphäre nur in starker Filterung erhalten, welche jedoch im Satellitenniveau ungeschwächt gemessen werden kann. Sie bewirkt - eventuell auf komplizierteren Umwegen über den Aufbau der verschiedenen Strahlungs- und Ionisationsgürtel sowie der Ozonschicht in und oberhalb der Stratosphäre - die verschiedenartigen Wetterabläufe in unserem abwechslungsreichen Klima, in welchem so viele verschiedene Gewächse gedeihen, obwohl es auf dem Meteorologenpfad oft den Anschein hat, als ob vorzugsweise Dornenhecken zu finden seien ...

\section{CLIMATOLOGIE ET MÉTÉOROLOGIE \\ AU DÉBUT DU DEUXIÈME SIÈCLE D’OBSERVATIONS RÉGULIÈRES EN SUISSE}

La climatologie et la synoptique accusent un développement constant depuis le début des observations faites régulièrement dans le réseau météorologique suisse, fondé le ler décembre 1863.

Un premier traité de climatologie, intitulé «Das Klima der Schweiz», de Maurer, Billwiller et Hess, a paru en 1909/10. Une nouvelle climatologie, basée sur les années 19o1-1960, va paraître dans le courant des années qui viennent. Elle comprendra 3 volumes: première partie: tabelles 
des différents éléments, deuxième partie: climatologie des diverses régions de la Suisse, troisième partie: climatologie des types de temps.

Cet ouvrage devrait être terminé en 1971 ; les méthodes les plus modernes (p. ex. cartes perforées) y seront utilisées. Le réseau des stations devient toujours plus malaisé à maintenir; il faudra songer à automatiser, ce qui n'est cependant pas facile pour quelques-uns des éléments. Pour la synoptique, des méthodes ont été développées, qui permettent de calculer les cartes de courants en altitude 24 à 96 heures à l'a vance. La prévision à moyenne et à longue échéance est cependant encore peu avancée. De nombreuses recherches seront encore nécessaires pour en améliorer les résultats.

\title{
DER GEIRANGERFJORD
}

\author{
OSKAR BÄR
}

Mit Farbbild

Kaum anderswo durchdringen sich Land und Meer so innig wie im südwestlichen Norwegen. Zwischen dem 59. und dem 63. Breitengrad stößt die See in rund einem Dutzend markantester Fjorde tief ins Land hinein vor. Der in unserer Farbtafel abgebildete Geirangerfjord, einer der nördlichsten in der berühmten Gruppe, zählt zu den schönsten und bekanntesten.

Die Fahrt dorthin führt vom Bredsund, etwas südlich des Hafenstädtchens Aalesund $\left(62^{\circ} 28^{\prime} \mathrm{n}\right.$. Br. $)$, vorerst in den Storfjord. Dieser anfänglich 4 bis $6 \mathrm{~km}$ breite Fjord weist ziemlich genau nach Osten, wo er nach rund $50 \mathrm{~km}$ scharf südwärts abbiegt und sich nach weitern $25 \mathrm{~km}$ verzweigt. Die beiden Arme, der Nordalsfjord (ostwärts) und der Sunelvsfjord (25 km südwärts bis Hellesylt) sind wunderbar schmale, von 1000 $1500 \mathrm{~m}$ hohen Felswänden eingefaßte Wasseradern. Im rechten Winkel zum Sunelvsfjord erstreckt sich der Geirangerfjord in sanfter Wellenlinie $15 \mathrm{~km}$ nach Osten und endet, sich zwischen steilen Wänden auf $800-1000$ m verengend, in Geiranger. In unserem Bild umfährt das Motorschiff «Oslofjord» die letzte Biegung, um nach etwa $5 \mathrm{~km}$ dieses Ziel zu erreichen.

Die Fjordlandschaft vermag uns stets aufs neue zu begeistern, und immer wieder taucht die Frage nach ihrer Entstehung auf. Fließendes Wasser und Gletschereis haben sich in die Erosionsarbeit geteilt. Die schon voreiszeitlich im Granit und Gneis des abgetragenen Kaledonischen Gebirges angelegten Flußtäler wurden zur Eiszeit umgeformt. In schmalen Rinnen konzentriertes und deshalb sehr rasch abfließendes Inlandeis leistete die Hauptarbeit. Je enger diese Abflußdüsen beschaffen waren, um so wirkungsvoller wurden sie vertieft, meistens bis rund $500 \mathrm{~m}$ unter den Meeresspiegel. Der $180 \mathrm{~km}$ lange Sognefjord hat in der Mitte sogar eine Tiefe von $1240 \mathrm{~m}$. Am Fjordausgang reicht aber eine Felsschwelle bis $100 \mathrm{~m}$ unter die Wasseroberfläche - ein Hinweis auf die entscheidende Wirkung der Gletschererosion. Wenn man bei uns die Alpenrandseen, vor allem den Urner- und den Walensee, mit den norwegischen Fjorden vergleicht, sollte ein wesentlicher Unterschied nicht außer acht gelassen werden: während sich in den Alpen über den glazialen Trogtälern überall zackige, frostverwitterte Gräte aufschwingen, die nie unter dem Eis der alpinen Gletscher begraben waren, erwartet uns über den Fjordwänden nichts ähnliches.

Die im Hintergrund des Bildes noch erkennbare, leicht gewellte und teilweise mit Schnee bedeckte Hochfläche nennt man im Norwegischen «Fjeld» oder «Fjell». Kanten und Gräte wurden dort während der Vergletscherung unter der mächtigen skandinavi- 